Document downloaded from:

http://hdl.handle.net/10251/125578

This paper must be cited as:

Bonet Solves, JA.; Jorda Mora, E.; Rodríguez-Arenas, A. (2018). Mean ergodic multiplication operators on weighted spaces of continuous functions. Mediterranean Journal of Mathematics. 15(3):1:108-11:108. https://doi.org/10.1007/s00009-018-1150-8

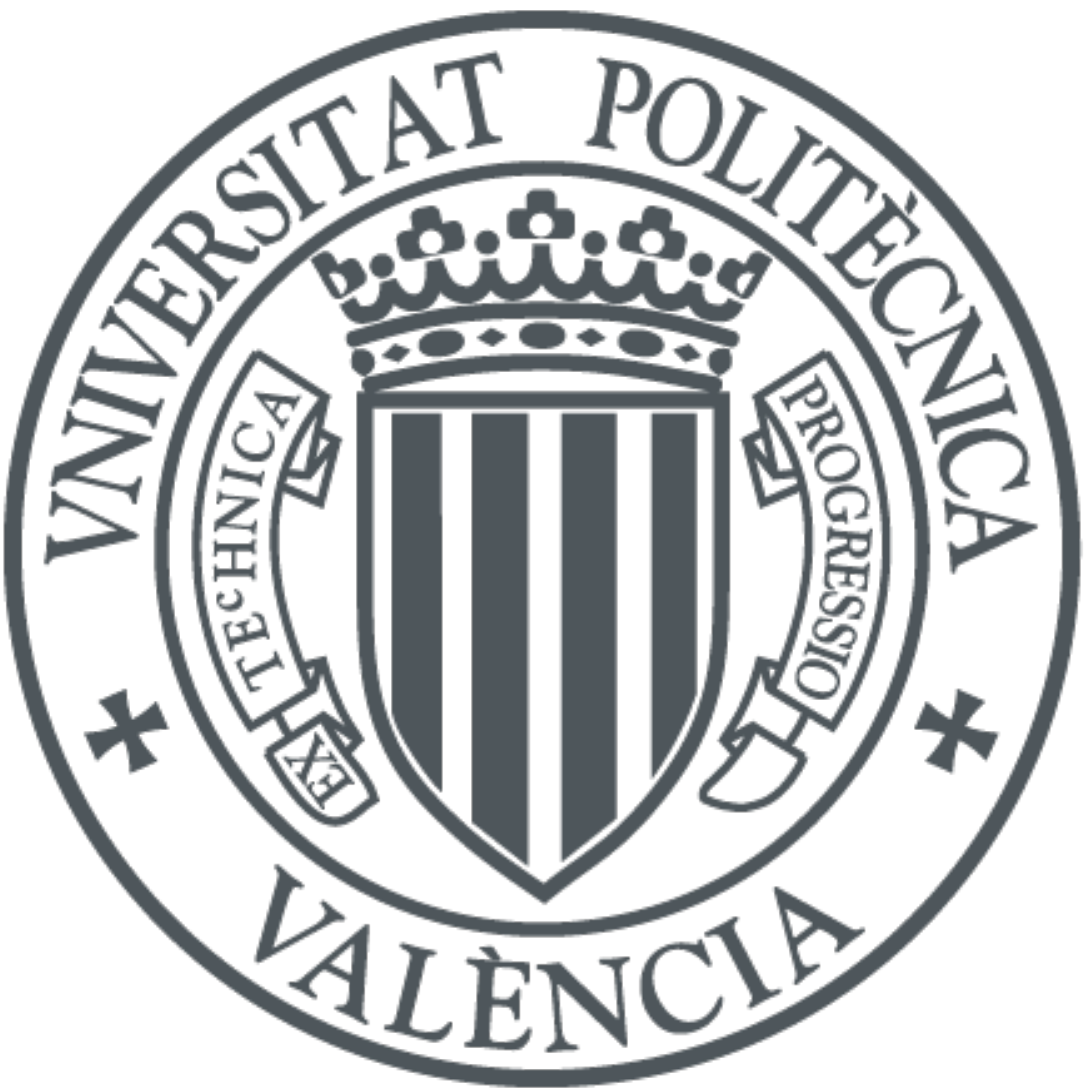

The final publication is available at

http://doi.org/10.1007/s00009-018-1150-8

Copyright Springer-Verlag

Additional Information 


\title{
Mean ergodic multiplication operators on weighted spaces of continuous functions
}

\author{
José Bonet, Enrique Jordá and Alberto Rodríguez
}

\begin{abstract}
Multiplication operators on weighted Banach spaces and locally convex spaces of continuous functions have been thoroughly studied. In this note we characterize when continuous multiplication operators on a weighted Banach space and on a weighted inductive limit of Banach spaces of continuous functions are power bounded, mean ergodic or uniformly mean ergodic. The behaviour of the operator on weighted inductive limits depends on the properties of the defining sequence of weights and it differs from the Banach space case.
\end{abstract}

\section{Introduction, Notation, and Motivation}

Multiplication operators on weighted spaces of (vector valued) continuous functions have been investigated by Manhas [13], [14], Singh and Manhas [15], [19], [21], Oubbi [17], [18] and Klilou and Oubbi [9] among others. See also the book by Singh and Manhas [20]. However, it seems that power boundedness and mean ergodicity of multiplication operators on weighted Banach spaces and weighted inductive limits of spaces of continuous functions had not been investigated. The case of multiplication operators on weighted spaces of analytic functions on the complex unit disc was studied by Bonet and Ricker in [8]. Many tools used in that paper, like the maximum modulus principle and certain properties of the Banach spaces of analytic functions, are not available in the case of continuous functions. Thus a different approach is needed. Our aim is to characterize when continuous multiplication operators on a weighted Banach space or on a weighted inductive limit of Banach spaces of continuous functions are power bounded, mean ergodic or uniformly mean ergodic. Our main results are Theorems 2.6 and 2.7 for operators on Banach spaces and Theorem 3.7 and Corollary 3.9 for operators on weighted inductive limits. Several examples show that the characterization of uniformly mean ergodic operators for weighted inductive limits does not coincide with the Banach space case.

2010 Mathematics Subject Classification. Primary: 47B38; 46E10; 46E15

Key words and phrases. Banach spaces of continuous functions, weighted inductive limits, multiplication operator, mean ergodic operator. 
We refer the reader to [22] for undefined terminology about set topology. We fix the notation for weighted spaces of continuous functions. In the sequel $X$ denotes a Hausdorff, normal, locally compact topological space. For example $X$ might be an open subset of $\mathbb{R}^{n}$ or a discrete space, i.e. and index set. The space of continuous functions from $X$ to $\mathbb{C}$ is denoted by $C(X)$ and the locally convex topology on $C(X)$ of pointwise convergence is denoted by $\tau_{p}$. We say $v \in C(X)$ is a weight if it is strictly positive. A function $f \in C(X)$ vanishes at infinity if for every $\varepsilon>0$ there exists a compact set $K \subset X$ such that $|f(x)|<\varepsilon$ if $x \in X \backslash K$. The weighted Banach spaces of continuous functions are defined by

$$
\begin{gathered}
C_{v}:=C_{v}(X)=\left\{f \in C(X):\|f\|_{v}:=\sup _{x \in X} v(x)|f(x)|<\infty\right\} \\
C_{v}^{0}:=C_{v}^{0}(X)=\{f \in C(X): v f \text { vanishes at infinity }\}
\end{gathered}
$$

equipped with the norm $\|\cdot\|_{v}$. In case $X$ is a discrete space, then we use the notation $\ell_{\infty}(v)=C_{v}$ and $c_{0}(v)=C_{v}^{0}[7]$. Clearly $C_{v}^{0} \subset C_{v}$ and every continuous function with compact support on $X$ is contained in $C_{v}^{0}$. Note that for $v \equiv 1, C_{v}$ coincides with the Banach space $C B(X)$ of bounded continuous functions endowed with the supremum norm $\|\cdot\|_{\infty}$, and $C_{v}^{0}$ is the space $C_{0}(X)$ of continuous functions vanishing at infinity, also endowed with the supremum norm. Given $\varphi \in C(X)$, the multiplication operator $M_{\varphi} \in \mathcal{L}(C(X))$ is defined by $M_{\varphi}: f \mapsto \varphi f$.

Our notation for functional analysis, locally convex spaces and inductive limits is standard. We refer the reader to [1], [16] and [23]. All the locally convex spaces are assumed to be Hausdorff. The weak topology of a locally convex Hausdorff space $E$ is denoted by $\sigma\left(E, E^{\prime}\right)$, where $E^{\prime}$ is the topological dual space of $E$. The space of continuous linear operators from $E$ into itself is denoted by $\mathcal{L}(E)$. We write $\mathcal{L}_{s}(E)$ and $\mathcal{L}_{b}(E)$ to denote $\mathcal{L}(E)$ when it is equipped with its strong operator topology and with the topology of uniform convergence on bounded sets of $E$, respectively. The Cesàro means of an operator $T \in \mathcal{L}(E)$ are defined by

$$
T_{[n]}:=\frac{1}{n} \sum_{m=1}^{n} T^{m}, \quad n \in \mathbb{N} .
$$

The operator $T$ is mean ergodic (resp. uniformly mean ergodic) if the sequence $\left\{T_{[n]}\right\}_{n=1}^{\infty}$ converges in $\mathcal{L}_{s}(X)$ (resp. in $\mathcal{L}_{b}(X)$ ). We say $T$ is power bounded if $\left\{T^{n}\right\}_{n=1}^{\infty}$ is an equicontinuous set of $\mathcal{L}(X)$. The standard text for mean ergodic operators in Banach spaces is [10]; see also [23].

\section{Multiplication operators on weighted Banach spaces of continuous functions}

In this section the multiplication operator $M_{\varphi}$ on $C_{v}$ and $C_{v}^{0}$ is denoted by $T_{\varphi}:=\left.M_{\varphi}\right|_{C_{v}}$ : $C_{v}(X) \longrightarrow C(X)$ and $S_{\varphi}:=\left.M_{\varphi}\right|_{C_{v}^{0}}: C_{v}^{0}(X) \longrightarrow C(X)$ respectively. The following result is well-known. More general results can be seen in [17, Theorem 4], [19, Theorem 2.1] and [21, Theorem 3.1] for example. 
Proposition 2.1 (i) Let $v, w$ be weights. Then $M_{\varphi}: C_{v} \longrightarrow C_{w}$ is continuous if and only if $\frac{w}{v} \varphi$ is bounded if and only if $M_{\varphi}: C_{v}^{0} \longrightarrow C_{w}^{0}$ is continuous. Moreover, $\left\|M_{\varphi}\right\|=$ $\sup _{x \in X} \frac{w(x)}{v(x)}|\varphi(x)|$ in both cases.

(ii) $T_{\varphi} \in \mathcal{L}\left(C_{v}\right)$ if and only if $S_{\varphi} \in \mathcal{L}\left(C_{v}^{0}\right)$ if and only if $\varphi$ is bounded. Moreover,

$$
\left\|T_{\varphi}\right\|_{v}=\left\|S_{\varphi}\right\|_{v}=\|\varphi\|_{\infty} .
$$

Lemma 2.2 Let $E$ be a locally convex space containing the functions with compact support on $X$ such that $E$ is continuously included in $\left(C(X), \tau_{p}\right)$. Assume that $M_{\varphi} \in \mathcal{L}(E)$. If $\lim _{n}\left(M_{\varphi}^{n} f\right)(x) / n=0$ for every $f \in E$, then $|\varphi(x)| \leq 1$. In particular if $M_{\varphi}$ is either power bounded or mean ergodic, then $\|\varphi\|_{\infty} \leq 1$.

Proof. If $M_{\varphi}$ is power bounded or mean ergodic, then $\lim _{n}\left(M_{\varphi}^{n} f\right)(x) / n=0$ for every $f \in E$ and every $x \in X$. Given $x \in X$, select a continuous function $g$ with compact support such that $g(x)=1$. We have $0=\lim _{n}\left|\left(M_{\varphi}^{n} g\right)(x)\right| / n=\lim _{n}|\varphi(x)|^{n} / n$. This yields $|\varphi(x)| \leq 1$.

Corollary 2.3 Let $\varphi \in C B(X)$. Then, $T_{\varphi} \in \mathcal{L}\left(C_{v}\right)$ is power bounded if and only if $S_{\varphi} \in \mathcal{L}\left(C_{v}^{0}\right)$ is power bounded if and only if $\|\varphi\|_{\infty} \leq 1$.

Remark 2.4 Let $E$ be a locally convex space containing the functions with compact support on $X$ such that $E$ is continuously included in $\left(C(X), \tau_{p}\right)$ and assume that $M_{\varphi} \in$ $\mathcal{L}(E)$. For each $f \in E$ and $n \in \mathbb{N}$ we have

$$
\left(\left(M_{\varphi}\right)_{[n]} f\right)(x)=\frac{f(x)}{n} \sum_{m=1}^{n}(\varphi(x))^{m}, \quad x \in X,
$$

and

$$
\left(\left(M_{\varphi}\right)_{[n]} f\right)(x)=\frac{f(x) \varphi(x)}{n} \cdot \frac{1-(\varphi(x))^{n}}{1-\varphi(x)}, \quad x \in X \backslash \varphi^{-1}(1) .
$$

In the case $\|\varphi\|_{\infty} \leq 1$, these formulas imply the convergence of $\left(M_{\varphi}\right)_{[n]} f$ uniformly on the compact subsets of $X$ to the (not necessarily continuous) function $h_{f}$ defined by

$$
h_{f}(x)= \begin{cases}f(x) & \text { if } \varphi(x)=1 \\ 0 & \text { if } \varphi(x) \neq 1\end{cases}
$$

Proposition 2.5 If $E$ is a locally convex space containing the functions with compact support on $X$ such that $E$ is continuously included in $\left(C(X), \tau_{p}\right)$. If $M_{\varphi} \in \mathcal{L}(E)$ and it is mean ergodic, then $\varphi^{-1}(1)$ is open.

Proof. By Lemma 2.2, $\|\varphi\|_{\infty} \leq 1$. Now suppose $\varphi^{-1}(1)$ is not open, then there exists $x \in X$ such that $\varphi(x)=1$ but it is not in the interior of $\varphi^{-1}(1)$. Therefore, for each $U \subset X$ open with $x \in U$, there exists $x_{U} \in U$ with $\varphi\left(x_{U}\right) \neq 1$. These $x_{U}$ form a net converging to $x$. Select a continuous function $g$ with compact support such that $g(x)=1$. By assumption 
$g \in E$. Since $M_{\varphi}$ is mean ergodic, the function $h_{g}$ defined in Remark 2.4 is continuous. However, $h_{g}(x)=1$ and $h_{g}\left(x_{U}\right)=0$, for every $U$, which is a contradiction, and $\varphi^{-1}(1)$ is open.

Theorem 2.6 Let $\varphi \in C B(X)$. Then, $S_{\varphi} \in \mathcal{L}\left(C_{v}^{0}\right)$ is mean ergodic if and only if $\|\varphi\|_{\infty} \leq$ 1 and $\varphi^{-1}(1)$ is open.

Proof. If $S_{\varphi}$ is mean ergodic, the conclusion follows from Lemma 2.2 and Proposition 2.5. To prove the converse, assume that $\|\varphi\|_{\infty} \leq 1$ and $Z:=\varphi^{-1}(1)$ is open. Hence, $Z$ and $X \backslash Z$ are both open and closed and thus $C_{v}^{0}(X)=C_{v}^{0}(Z) \oplus C_{v}^{0}(X \backslash Z)$. Since the restriction of $S_{\varphi}$ to the first space is the identity operator, we may assume $\varphi \neq 1$ in $X$. From the formula (2.2) it follows that $\left(v\left(S_{\varphi}\right)_{[n]} f\right)_{n}$ is pointwise convergent to 0 for each $f \in C_{v}^{0}$ (even for the compact topology). If $\widehat{X}$ is the Alexandroff compactification of $X$, the isometry $C_{v}^{0}(X) \hookrightarrow C(\widehat{X}), f \mapsto v f$, together with Riesz's representation theorem yield that actually $\left(\left(S_{\varphi}\right)_{[n]} f\right)_{n}$ is weakly convergent to 0 for every $f \in C_{v}^{0}$. Now, since $S_{\varphi}$ is power bounded, it follows from the classical mean ergodic theorem (see the equivalence of (ii) and (iii) of Theorem 1.1 in [10, page 72]) that $S_{\varphi}$ is mean ergodic.

Theorem 2.7 Let $\varphi \in C B(X)$. The following assertions are equivalent:

(1) $T_{\varphi} \in \mathcal{L}\left(C_{v}\right)$ is uniformly mean ergodic.

(2) $S_{\varphi} \in \mathcal{L}\left(C_{v}^{0}\right)$ is uniformly mean ergodic.

(3) $\|\varphi\|_{\infty} \leq 1, \inf \left\{|\varphi(x)-1|: x \in X \backslash \varphi^{-1}(1)\right\}>0$ and $\varphi^{-1}(1)$ is open.

(4) $T_{\varphi} \in \mathcal{L}\left(C_{v}\right)$ is mean ergodic.

Proof. Clearly (1) implies (2) and (4).

To see that (2) implies (3) suppose that $S_{\varphi}$ is uniformly mean ergodic. Then, $S_{\varphi}$ is mean ergodic and by Theorem 2.6, $\|\varphi\|_{\infty} \leq 1$ and $\varphi^{-1}(1)$ is open. Thus, as in the proof of Theorem 2.6, we may assume $\varphi \neq 1$ in $X$. In that case it is easy to see that $I-S_{\varphi}$ is injective. Since $S_{\varphi}$ is uniformly mean ergodic, we can apply a theorem by Lin [11] (see also $\left[10\right.$, Theorem 2.1]) to conclude that that $I-S_{\varphi}$ is also surjective. The continuous operator $I-S_{\varphi}$ is bijective if and only if $\frac{1}{1-\varphi}$ is bounded. This implies $\inf \{|\varphi(x)-1|: x \in X\}>0$.

We show that (3) implies (1). Since $\varphi^{-1}(1)$ is open, it is enough to consider the case $\varphi \neq 1$ in $X$. The assumption (3) and the formula (2.2) imply that there is $C>0$ such that for each $f \in C_{v}$ and each $n \in \mathbb{N}$ we have $\left\|\left(T_{\varphi}\right)_{[n]} f\right\|_{v} \leq C\|f\|_{v} / n$. Thus, $\left\|\left(T_{\varphi}\right)_{[n]}\right\| \rightarrow 0$ as $n \rightarrow \infty$, and $T_{\varphi}$ is uniformly mean ergodic.

Finally we prove that (4) implies (3). Since $T_{\varphi}$ is mean ergodic, also $S_{\varphi}$ is mean ergodic and then $\|\varphi\|_{\infty} \leq 1$ and $\varphi^{-1}(1)$ is open. Again we can restrict ourselves to the case $\varphi \neq 1$ in $X$. Let $\beta X$ be the Stone-Cech compactification of $X$ and denote by $\widehat{g}$ the unique extension of a function $g \in C B(X)$ to $\beta X$. Consider the isomorphisms $C_{v}(X) \longrightarrow C B(X)$, $f \mapsto v f$ and $C B(X) \longrightarrow C(\beta X), h \mapsto \widehat{h}$. Also note that, since $\beta X$ is compact, $C(\beta X)$ 
coincides with the space of functions vanishing at infinity $C_{0}(\beta X)$. Thus we have an isomorphism between $C_{v}(X)$ and $C_{0}(\beta X)$. Apply Theorem 2.6 to $S_{\widehat{\varphi}} \in \mathcal{L}\left(C_{0}(\beta X)\right)$ to find that $(\widehat{\varphi})^{-1}(1)$ is open in $\beta X$. Since we have assumed that $\varphi \neq 1$ in $X$, we must have $(\widehat{\varphi})^{-1}(1) \subset \beta X \backslash X$. But $\beta X \backslash X$ has empty interior and thus $(\widehat{\varphi})^{-1}(1)=\emptyset$. From this follows that $\inf _{x \in X}|1-\varphi(x)|>0$.

Remark 2.8 If the space $X$ is discrete, then $C_{v}=l_{\infty}(v)$ is a Grothendieck Banach space with the Dunford-Pettis property. In this case a theorem of Lotz [12] implies that $T_{\varphi}$ is mean ergodic if and only if it is uniformly mean ergodic. This argument cannot be used to prove Theorem 2.7 in full generality, since not all $C_{v}(X)$ spaces have the GDP property. It is well known that if $K$ is a compact space then $C(K)$ is a Grothendieck space if and only if $K$ is extremely disconnected if and only if $c_{0}$ is not complemented in $C(K)$.

It is now easy to exhibit examples of multiplication operators that are mean ergodic but not uniformly mean ergodic: Define $\varphi: \mathbb{N} \rightarrow \mathbb{N}$ as $\varphi(2 n)=1, \varphi(2 n+1)=(1-1 / n)$. For every weight $v$ on $\mathbb{N}$ we have that $S_{\varphi}: c_{0}(v) \rightarrow c_{0}(v),\left(a_{n}\right) \mapsto\left(\varphi(n) a_{n}\right)$ is mean ergodic by Theorem 2.6 but not uniformly mean ergodic by Theorem 2.7 and $T_{\varphi}: l_{\infty}(v) \rightarrow l_{\infty}(v)$, $\left(a_{n}\right) \mapsto\left(\varphi(n) a_{n}\right)$ is not mean ergodic by Theorem 2.7 .

\section{Multiplication operators on weighted inductive lim- its of spaces of continuous functions}

Weighted inductive limits of spaces of continuous and holomorphic functions have been thoroughly investigated since the seminal work of Bierstedt, Meise and Summers [6] and [7]; see [1], [2], [3], [5] and the references therein. In this section we characterize power bounded and (uniformly) mean ergodic multiplication operators defined on weighted inductive limits of spaces of continuous functions defined on a normal locally compact Hausdorff topological space $X$.

Throughout this section $V=\left(v_{k}\right)_{k}$ denotes a decreasing sequence of continuous strictly positive weights. The weighted inductive limits associated to $V$ are $V C=V C(X):=$ $\operatorname{ind}_{k} C_{v_{k}}$ and $V_{0} C=V_{0} C(X):=\operatorname{ind}_{k} C_{v_{k}}^{0}$. They are Hausdorff (LB)-spaces and $V_{0} C \subset V C$ is a topological subspace of $V C$ by [6, Theorem 1.3]. In case $X$ is discrete, these spaces are precisely Köthe co-echelon spaces of order infnity and zero, [7].

The Nachbin family associated to $V$ is

$$
\begin{gathered}
\bar{V}:=\{\bar{v}: X \longrightarrow(0, \infty): \bar{v} \text { is upper semicontinuous and for each } k \in \mathbb{N}, \\
\left.\frac{\bar{v}}{v_{k}} \text { is bounded on } X\right\} .
\end{gathered}
$$

The weighted spaces of continuous functions associated with $V C$ and $V_{0} C$ are defined as follows.

$$
C \bar{V}=\left\{f \in C(X): p_{\bar{v}}(f):=\sup _{x \in X} \bar{v}(x)|f(x)|<\infty, \forall \bar{v} \in \bar{V}\right\} .
$$




$$
C \bar{V}_{0}=\{f \in C(X): \bar{v} f \text { vanishes at infinity, } \forall \bar{v} \in \bar{V}\} .
$$

They are endowed with the locally convex topology generated by the seminorms $p_{\bar{v}}, \bar{v} \in \bar{V}$. It is well known that $V C=C \bar{V}$ algebraically and the topology in $C \bar{V}$ is in general coarser, but they share bounded sets; in fact every bounded subset of $C \bar{V}$ is contained and bounded in a step $C_{v_{n}}$. Moreover, $C \bar{V}_{0}$ is a closed subspace of $C \bar{V}$ and $V_{0} C$ is a topological subspace of $C \bar{V}_{0}$, hence of $V C$. We refer the reader to [6] for these results.

The characterization of continuous multiplication operators follows at once from Grothendieck's factorization theorem [16, Theorem 24.33] and Proposition 2.1.

Proposition 3.1 The following assertions are equivalent:

1. $M_{\varphi}: V C(X) \longrightarrow V C(X)$ is continuous.

2. For every $k$ there exists $l$ such that $M_{\varphi}: C_{v_{k}}(X) \longrightarrow C_{v_{l}}(X)$ is continuous.

3. For every $k$ there exists $l$ such that $\frac{v_{l}}{v_{k}} \varphi$ is bounded on $X$,

4. $M_{\varphi}: V_{0} C(X) \longrightarrow V_{0} C(X)$ is continuous,

5. for every $k$ there exists $l$ such that $M_{\varphi}: C_{v_{k}}^{0}(X) \longrightarrow C_{v_{l}}^{0}(X)$ is continuous.

Proposition $3.2 M_{\varphi} \in \mathcal{L}(V C)$ is power bounded if, and only if, $M_{\varphi} \in \mathcal{L}\left(V_{0} C\right)$ is power bounded, if and only if $\|\varphi\|_{\infty} \leq 1$.

Proof. The necessity of $\|\varphi\|_{\infty} \leq 1$ for both $V C$ and $V_{0} C$ follows from Lemma 2.2. For the sufficiency assume $\|\varphi\|_{\infty} \leq 1$. Since both $V_{0} C$ and $V C$ are barrelled spaces, by Banach Steinhaus theorem it is enough to show that every orbit $\left(\left(M_{\varphi}\right)^{n} f\right)_{n}$ is bounded for each $f$ in the space. Fix $f \in V_{0} C$. There exist $k$ and $\lambda>0$ such that $f \in \lambda B_{k}$, where $B_{k}$ is the closed unit ball of $C_{v_{k}}$. This implies $\left(M_{\varphi}\right)^{n} f \in \lambda B_{k} \cap V_{0} C$ for each $n$. Now it is enough to keep in mind that $\left(k B_{k} \cap V_{0} C\right)_{k}$ is a fundamental system of bounded sets of $V_{0} C$ by [6, Theorem 1.3]. The case of $V C$ is similar.

Proposition 3.3 $M_{\varphi} \in \mathcal{L}\left(V_{0} C\right)$ is mean ergodic if and only if $\|\varphi\|_{\infty} \leq 1$ and $\varphi^{-1}(1)$ is open.

Proof. If $M_{\varphi} \in \mathcal{L}\left(V_{0} C\right)$ is mean ergodic, then by Lemma 2.2 and Proposition 2.5, we get the necessary conditions.

Conversely, if $\|\varphi\|_{\infty} \leq 1$ and $\varphi^{-1}(1)$ is open, then $S_{\varphi} \in \mathcal{L}\left(C_{v_{k}}^{0}\right)$ is mean ergodic for every $k \in \mathbb{N}$ by Theorem 2.6. By the properties of inductive limits, it follows that $M_{\varphi} \in \mathcal{L}\left(V_{0} C\right)$ is mean ergodic.

The next technical lemma was proved in [4, Prop.3] and it is very useful in our setting.

Lemma 3.4 There is a fundamental system $\mathcal{U}$ of neighbourhoods of 0 for $V C$ such that if $U \in \mathcal{U}$ and $f \in U$, then, for every $g \in V C$ with $|g| \leq|f|$, one gets $g \in U$. 
Proposition 3.5 The following assertions are equivalent:

(1) $M_{\varphi} \in \mathcal{L}(V C)$ is mean ergodic.

(2) $M_{\varphi} \in \mathcal{L}(V C)$ is uniformly mean ergodic.

Furthermore, if these equivalent conditions hold, then $\|\varphi\|_{\infty} \leq 1, \varphi^{-1}(1)$ is open, $h_{f}$ as in (2.3) belongs to $V C$ for each $f \in V C$, and $\left(M_{\varphi}\right)_{[n]} \rightarrow P$ in $\mathcal{L}_{b}(V C)$, where $P \in \mathcal{L}(V C)$ with $P(f)=h_{f}$.

Proof. It suffices to show that $M_{\varphi}$ is uniformly mean ergodic whenever it is mean ergodic. Let $B_{k}$ denote the closed unit ball of $C_{v_{k}}$. By [6] every bounded set of $V C$ is contained in a multiple of some $B_{k}$. Since $M_{\varphi}$ is mean ergodic, $h_{g}$ belongs to $V C$ for each $g \in V C$. Fix $k$. We have to show that $\left(M_{\varphi}\right)_{[n]} g-h_{g}$ converges to 0 uniformly on $g \in B_{k}$. Set $f_{k}:=1 / v_{k}$. If $U \in \mathcal{U}$, where $\mathcal{U}$ is the basis of neighbourhoods of $V C$ in Lemma 3.4, there exists $n_{0}$ such that for $n \geq n_{0},\left(M_{\varphi}\right)_{[n]} f_{k}-h_{f_{k}} \in U$, since $M_{\varphi}$ is mean ergodic. Every $g \in B_{k}$ satisfies $|g| \leq f_{k}$, then a simple computation and Lemma 3.4 yields $\left(M_{\varphi}\right)_{[n]} g-h_{g} \in U$, for $n \geq n_{0}$.

The consequences of conditions (1) and (2) in the statement now follow from arguments in Lemma 2.2, Remark 2.4 and Proposition 2.5.

Although $M_{\varphi}$ is mean ergodic if and only if it is uniformly mean ergodic on $V C$, these conditions do not necessarily imply $\inf \left\{|\varphi(x)-1|: x \in X \backslash \varphi^{-1}(1)\right\}>0$. Compare with Theorem 2.7. The first examples can be obtained assuming a condition on the sequence $V$.

The sequence $V$ satisfies condition $(S)$ if for each $n$ there exists $m>n$ such that $v_{m} / v_{n}$ vanishes at infinity. It is easy to see that condition (S) holds if and only if $V C=V_{0} C$. For example, the sequence $V=\left(v_{n}\right)_{n}$ of weights $v_{n}(z):=(1-|z|)^{n}, z \in \mathbb{D}, n \in \mathbb{N}$, on the complex unit disc $\mathbb{D}$ satisfies condition $(\mathrm{S})$. We refer the reader to [6] for the relevance and more examples of condition (S). In the context of Köthe echelon and co-echelon spaces condition (S) characterizes the property of being Schwartz [7]. The next corollary is a consequence of Propositions 3.3 and 3.5.

Corollary 3.6 Assume that the sequence $V$ satisfies condition $(S)$. The following conditions are equivalent for $M_{\varphi} \in \mathcal{L}(V C)$ :

(1) $M_{\varphi} \in \mathcal{L}(V C)$ is uniformly mean ergodic.

(2) $M_{\varphi} \in \mathcal{L}(V C)$ is mean ergodic.

(3) $\|\varphi\|_{\infty} \leq 1$ and $\varphi^{-1}(1)$ is open.

Now we characterize when $M_{\varphi}$ is uniformly mean ergodic on $V_{0} C$.

Theorem 3.7 The following assertions are equivalent:

(1) $M_{\varphi} \in \mathcal{L}\left(V_{0} C\right)$ is uniformly mean ergodic. 
(2) $\|\varphi\|_{\infty} \leq 1, \varphi^{-1}(1)$ is open in $X$ and for every $k \in \mathbb{N}$ and every $\bar{v} \in \bar{V}$,

$$
\lim _{n \rightarrow \infty} \sup _{x \in Y} \frac{1}{n} \frac{\bar{v}(x)}{v_{k}(x)}|\varphi(x)| \frac{\left|1-\varphi(x)^{n}\right|}{|1-\varphi(x)|}=0,
$$

where $Y=X \backslash \varphi^{-1}(1)$.

Proof. It is enough to prove the result when $\varphi(x) \neq 1$ for each $x \in X$. Thus we assume $X=Y$. We set, for each $k, n \in \mathbb{N}, \bar{v} \in \bar{V}$ and $x \in X$,

$$
R_{n}^{k, \bar{v}}(x):=\frac{1}{n} \frac{\bar{v}(x)}{v_{k}(x)}|\varphi(x)| \frac{\left|1-\varphi(x)^{n}\right|}{|1-\varphi(x)|} .
$$

Assume first that condition (2) holds. Fix $k \in \mathbb{N}$. For each $f \in V_{0} C$ such that $|f| \leq 1 / v_{k}$ and each $\bar{v} \in \bar{V}$ we have

$$
\bar{v}(x)\left|\left(M_{\varphi}\right)_{[n]} f(x)\right| \leq R_{n}^{k, \bar{v}}(x)
$$

for each $n \in \mathbb{N}$ and each $x \in X$. Given $\varepsilon>0$ we apply (2) to find $n(0) \in \mathbb{N}$ such that $R_{n}^{k, \bar{v}}(x)<\varepsilon$ for each $x \in X$ and each $n \geq n(0)$. Hence $\bar{v}(x)\left|\left(M_{\varphi}\right)_{[n]} f(x)\right|<\varepsilon$ for each $x \in X, n \geq n(0)$ and each $f \in V_{0} C$ such that $|f| \leq 1 / v_{k}$. By [6, Theorem 1.3] $V_{0} C$ is a topological subspace of $C \bar{V}_{0}$ and every bounded set of $V_{0} C$ is contained in a multiple of $\left\{f \in V_{0} C:|f| \leq 1 / v_{k}\right\}$ for some $k$. Therefore $\left(\left(M_{\varphi}\right)_{[n]}\right)_{n}$ converges to 0 in $\mathcal{L}_{b}\left(V_{0} C\right)$, and $M_{\varphi}$ is uniformly mean ergodic.

To prove the converse, suppose that $M_{\varphi} \in \mathcal{L}\left(V_{0} C\right)$ is uniformly mean ergodic. Fix $k \in \mathbb{N}$ and $\bar{v} \in \bar{V}$. Given $\varepsilon>0$ there is $n(0) \in \mathbb{N}$ such that $\bar{v}(x)\left|\left(M_{\varphi}\right)_{[n]} f(x)\right|<\varepsilon$ for each $x \in X, n \geq n(0)$ and each $f \in V_{0} C$ such that $|f| \leq 1 / v_{k}$. Now, for an arbitrary $z \in X$, there is a continuous function with compact support $h \in C(X)$ such that $0 \leq h \leq 1$ and $h(z)=1$. Then $g:=h / v_{k} \in V_{0} C$ and $|g| \leq 1 / v_{k}$ on $X$. This implies, for $n \geq n(0)$,

$$
R_{n}^{k, \bar{v}}(z)=\frac{\bar{v}(z)}{k}\left|\left(M_{\varphi}\right)_{[n]} g(z)\right|<\varepsilon .
$$

Since $z \in X$ is arbitrary, we have shown that $\lim _{n \rightarrow \infty} \sup _{z \in X} R_{n}^{k, \bar{v}}(z)=0$. The other statements in condition (2) follow from Proposition 3.3.

Corollary 3.8 If $\|\varphi\|_{\infty} \leq 1, \varphi^{-1}(1)$ is open in $X$ and for every $k \in \mathbb{N}$ there exists $l \geq k$ such that

$$
\lim _{n \rightarrow \infty} \sup _{x \in Y} \frac{1}{n} \frac{v_{l}(x)}{v_{k}(x)}|\varphi(x)| \frac{\left|1-\varphi(x)^{n}\right|}{|1-\varphi(x)|}=0,
$$

then $M_{\varphi} \in \mathcal{L}\left(V_{0} C\right)$ is uniformly mean ergodic.

Proof. This is a consequence of Theorem 3.7 since, for every $\bar{v} \in \bar{V}$ and each $l \in \mathbb{N}$, there exists $\alpha_{l}>0$ such that $\bar{v} \leq \alpha_{l} v_{l}$.

Corollary 3.9 Assume that $V C=C \bar{V}$ holds topologically. Then $M_{\varphi} \in \mathcal{L}(V C)$ is uniformly mean ergodic if and only if condition (2) of Theorem 3.7 holds. 
Proof. If $M_{\varphi} \in \mathcal{L}(V C)$ is uniformly mean ergodic, then $M_{\varphi} \in \mathcal{L}\left(V_{0} C\right)$ is uniformly mean ergodic, since $V_{0} C$ is a topological subspace of $V C$. We can apply Theorem 3.7 to conclude that condition (2) in this theorem holds. Conversely, as $V C=C \bar{V}$ algebraically and topologically, it is not hard to prove that condition (2) in Theorem 3.7 implies that the sequence $\left(\left(M_{\varphi}\right)_{[n]}\right)_{n}$ converges in $\mathcal{L}_{b}(V C)$ and $M_{\varphi} \in \mathcal{L}(V C)$ is uniformly mean ergodic.

The topological identity $V C=C \bar{V}$ was characterized by a condition (D) formulated in terms of the weights $V=\left(v_{n}\right)_{n}$ by Bastin, Bierstedt, Bonet and Vogt. See [2], [3] and [5].

Corollary 3.10 Assume that $\|\varphi\|_{\infty} \leq 1$ and $\varphi(x) \neq 1$ for each $x \in X$.

(i) If for all $k$ there is $l>k$ such that

$$
\sup _{x \in X} \frac{v_{l}(x)}{v_{k}(x)} \frac{1}{|1-\varphi(x)|}<\infty
$$

then $M_{\varphi} \in \mathcal{L}\left(V_{0} C\right)$ is uniformly mean ergodic.

(ii) If $\inf \{|1-\varphi(x)|: x \in X\}>0$, then $M_{\varphi} \in \mathcal{L}\left(V_{0} C\right)$ is uniformly mean ergodic.

Proof. The hypothesis of part (i) implies the assumption in Corollary 3.8. Part (ii) follows from (i).

Examples 3.11 We mention two examples of sequences $V=\left(v_{n}\right)_{n}$ which do not satisfy condition (S) and uniformly mean ergodic multiplication operators $M_{\varphi} \in L\left(V_{0} C\right)$ such that $\inf \left\{|1-\varphi(x)|: x \in X \backslash \varphi^{-1}(1)\right\}>0$ is not satisfied. These examples show that the converse of Corollary 3.10 does not hold and they should be compared with Theorem 2.7 and Corollary 3.6.

(1) Let $X=\mathbb{D}$ be the complex unit disc. Consider $V=\left(v_{k}\right)_{k}$ with $v_{k}(z)=\min \left(1,|1-z|^{k}\right)$. The sequence $V$ does not satisfy condition $(\mathrm{S})$, since $v_{k}(-1+a)=1$ for each $\left.a \in\right] 0,1[$. Take $\varphi(z)=z$. Clearly $\varphi(z) \neq 1$ for each $z \in \mathbb{D}$, but inf $\{|1-\varphi(x)|: x \in X\}=0$.

On the other hand, for any $n \in \mathbb{N}$ we have, for $|1-z| \leq 1$,

$$
\frac{v_{k+1}(z)}{v_{k}(z)} \cdot \frac{1}{|1-\varphi(z)|}=\frac{|1-\varphi(z)|^{k+1}}{|1-\varphi(z)|^{k+1}}=1
$$

and for $|1-z| \geq 1$,

$$
\frac{v_{k+1}(z)}{v_{k}(z)} \cdot \frac{1}{|1-\varphi(z)|}=\frac{1}{|1-z|} \leq 1
$$

We can apply Corollary 3.10 (i) to conclude that $M_{\varphi}$ is uniformly mean ergodic in $V_{0} C$.

(2) Let $X=\{z \in \mathbb{C}: \operatorname{Im} z>0\}$ be the upper half plane in $\mathbb{C}$. Define $v_{k}(z):=$ $\exp (-k \operatorname{Im} z), z \in X, k \in \mathbb{N}$, and $V=\left(v_{k}\right)_{k}$. It is easy to check that the sequence $V$ does 
not satisfy condition (S). The function $\varphi(z)=1-e^{i z}, z \in X$, satisfies $\varphi(z) \neq 1$ for each $z \in X$ and $\inf \{|1-\varphi(x)|: x \in X\}=0$.

We have

$$
\frac{v_{k+1}(z)}{v_{k}(z)} \cdot \frac{1}{|1-\varphi(z)|}=1
$$

Therefore $M_{\varphi}$ is uniformly mean ergodic in $V C$ by Corollary 3.10 (i).

(3) It is not hard to show that both sequences $V$ in the examples above are regularly decreasing in the sense of [6, Definition 2.1]. This implies that $V C=C \bar{V}$ holds topologically and the multiplication operators $M_{\varphi}$ are also uniformly mean ergodic on $V C$.

Acknowledgement. The research of Bonet was partially supported by Project Prometeo/2017/102 of the Generalitat Valenciana. The authors authors were also partially supported by MINECO Project MTM2016-76647-P. Rodríguez also thanks the support of the grant PAID-01-16 of the Universitat Politècnica de València.

\section{References}

[1] K.D. Bierstedt, An introduction to locally convex inductive limits Functional analysis and its applications (Nice, 1986), 35-133, ICPAM Lecture Notes, World Sci. Publishing, Singapore, 1988.

[2] K.D. Bierstedt, A survey of some results and open problems in weighted inductive limits and projective description for spaces of holomorphic functions, Bull. Soc. Roy. Sci. Liège 70 (2001), no. 4-6, 167-182.

[3] K.D. Bierstedt, J. Bonet, Some recent results on $\mathrm{VC}(\mathrm{X})$, Advances in the theory of Fréchet spaces (Istanbul, 1988), 181-194, NATO Adv. Sci. Inst. Ser. C Math. Phys. Sci., 287, Kluwer Acad. Publ., Dordrecht, 1989.

[4] K.D. Bierstedt, J. Bonet, Completeness of the (LB)-spaces VC(X), Arch. Math. (Basel) 56 (1991), no. 3, 281-285.

[5] K.D. Bierstedt, J. Bonet, Some aspects of the modern theory of Fréchet spaces, Rev. R. Acad. Cienc. Exactas Fís. Nat. Ser. A Mat. 97 (2003), no. 2, 159-188.

[6] K.D. Bierstedt, R. Meise, W.H. Summers, A projective description of weighted inductive limits, Trans. Amer. Math. Soc. 272 (1982), no. 1, 107-160.

[7] K.D. Bierstedt, R. Meise, W.H. Summers, Köthe sets and Köthe sequence spaces, Functional analysis, holomorphy and approximation theory (Rio de Janeiro, 1980), $27-91$.

[8] J. Bonet, W.J. Ricker, Mean ergodicity of multiplication operators in weighted spaces of holomorphic functions, Arch. Math. 92 (2009), 428-437. 
[9] M. Klilou, L. Oubbi, Multiplication operators on generalized weighted spaces of continuous functions, Mediterr. J. Math. 13 (2016), no. 5, 3265-3280.

[10] U. Krengel, Ergodic Theorems, de Gruyter, Berlin, 1985.

[11] M. Lin, On the uniform ergodic theorem, Proc. Am. Math. Soc., Vol.43, 2, April 1974.

[12] H.P. Lotz, Uniform convergence of operators on $L^{\infty}$ and similar spaces, Math. Z., 190 (1985), 207-220.

[13] J.S. Manhas, Compact multiplication operators on weighted spaces of vector-valued continuous functions, Rocky Mountain J. Math. 34 (2004), no. 3, 1047-1057.

[14] J.S. Manhas, Compact and weakly compact multiplication operators on weighted spaces of vector-valued continuous functions, Acta Sci. Math. (Szeged) 70 (2004), no. $1-2,361-372$.

[15] J.S. Manhas, R.K. Singh, Compact and weakly compact weighted composition operators on weighted spaces of continuous functions, Integral Equations Operator Theory 29 (1997), no. 1, 63-69.

[16] R. Meise, D. Vogt, Introduction to Functional Analysis, The Clarendon Press, Oxford University Press, New York, 1997.

[17] L. Oubbi, Multiplication operators on weighted spaces of continuous functions, Port. Math. (N.S.) 59 (2002), no. 1, 111-124.

[18] L. Oubbi, Weighted composition operators on non-locally convex weighted spaces, Rocky Mountain J. Math. 35 (2005), no. 6, 2065-2087.

[19] R.K. Singh, J.S. Manhas, Multiplication operators on weighted spaces of vector-valued continuous functions, J. Austral. Math. Soc. Ser. A 50 (1991), no. 1, 98-107.

[20] R.K. Singh, J.S. Manhas, Composition operators on function spaces, North-Holland Publishing Co., Amsterdam, 1993.

[21] R.K. Singh, J.S. Manhas, Operators and dynamical systems on weighted function spaces, Math. Nachr. 169 (1994), 279-285.

[22] A. Wilanski, Topology for Analysis, Ginn, Waltham, 1970.

[23] K. Yosida, Functional Analysis, Springer-Verlag, Berlin, Heidelberg, New York, 1980.

\section{Authors' addresses:}

Instituto Universitario de Matemática Pura y Aplicada IUMPA,

Universitat Politècnica de València,

E-46022 Valencia, Spain

E-mail: jbonet@mat.upv.es, ejorda@mat.upv.es, alrodar3@posgrado.upv.es 